\title{
38. SOME MAGNETIC PROPERTIES OF LEG 34 IGNEOUS ROCKS ${ }^{1}$
}

\author{
G. Tarasiewicz, E. Tarasiewicz, and C.G.A. Harrison, \\ University of Miami, Rosenstiel School of Marine and Atmospheric Science, Miami, Florida
}

\section{INTRODUCTION}

Twenty-four samples of igneous rock were sampled for study at the University of Miami. The original purpose was to determine paleolatitudes by measuring the inclination of magnetization, but it became clear that many were so unstable that little information could be obtained concerning the direction of the magnetic field during the time of formation of the rocks. Therefore, a study of the viscous remanent magnetization was made. This is a preliminary report of these studies.

\section{DEMAGNETIZATION}

The basalt cores were examined and described in a preliminary Hole summary book sent to all investigators receiving samples for this leg. Based on these preliminary descriptions, the textures of the basaltic rocks sent to the University of Miami ranged from diabasic to aphanitic. All samples were measured in a spinner magnetometer to determine their NRM. They were all run through a complete sequence of alternating field demagnetization at the following peak field strengths, given in oersteds $20,30,45,67,100,150,225$, $338,500,750$. A stability index was determined using the method of Tarling and Symons (1967). This involves the determination of the maximum value of the quantity $S$ where

$$
S=\frac{\sqrt{R}}{\theta^{\circ} 63}
$$

$\theta^{\circ}{ }_{63}$ is the circular standard deviation (Fisher, 1953) of three or more directions of magnetization obtained after demagnetization at consecutive higher demagnetizing field strengths (NRM being considered as demagnetizing in zero field strength). $R$ is the range of field values used. Higher values of $S$ indicate a smaller scatter of directions if $R$ is the same, and hence a greater stability. Figure 1 shows results from these demagnetization experiments. The top portion of the figure shows the range of demagnetizing field for which the maximum value of $S$ or the stability index. The divisions at the left of this portion of Figure 1 are the designations suggested by Tarling and Symons (1967). It can be seen that seven of the samples are classified as very stable, three of the samples are classified as stable, three of the samples as poorly stable, dour of the samples as metastable, and seven of the samples as unstable. There is a general tendency for the maximum value of $S$ to be obtained

\footnotetext{
'Contribution from the University of Miami, Rosenstiel School of Marine and Atmospheric Science.
}

over a larger range of demagnetizing field $(R)$ if the stability is low. For instance, there are five samples for which the maximum value of $S$ is obtained over the whole range of demagnetizing field values ( 0 to 750 oe) and four of these are classified as unstable and the other is classified as metastable.

Many of the samples described here come from the same levels as samples for which paleomagnetic measurements have been made and described in the Hole Summary Book sent to investigators of Leg 34 material. These paleomagnetic measurements generally involved demagnetization up to 100 to 200 oe. It was thus possible to calculate values of $S$ for these measurements and to compaare them with the values of $S$ determined at the University of Miami. Although many of the $S$ values are similar, there are several large discrepancies. Sample 319A-2-1, 106-109 cm gave a value of $S$ of 0.619 in the Hole Summary Book for demagnetization to $167 \mathrm{oe}$, and a value of 5.17 for demagnetization up to 150 oe in this study. The maximum value of $S$ for this sample is 7.3 for demagnetization between 67 and 150 oe. Sample 319A-3-2, 108-111 $\mathrm{cm}$ gave a value of $S$ of 0.382 for demagnetization up to 200 oe in the initial preprint and a value of 4.48 for demagnetization up to 225 oe in this study. Six of the samples whose paleomagnetic results were quoted in the Hole Summary Book gave much higher stabilities than they did in the results of these studies. These samples are shown in Table 1. Of these six samples, two gave maximum values of $S$ which were close to the values of $S$ derived from the initial preprint. Sample 319A-6-1, 142$145 \mathrm{~cm}$ gave a maximum value of $S$ of 3.75 for demagnetization between 150 and 338 oe and Sample 320B-3-1, 76-79 $\mathrm{cm}$ gave a maximum value of $S$ of 8.5 for demagnetization between 67 and 150 oe.

We see that this study sometimes gives much higher stabilities than did the shipboard study, and sometimes gives much lower stabilities. The reason for this is unclear but it may be due to different storage histories of the two samples taken from the same level.

Figure 1 also shows the values of the NRM intensity given in micro emu/cc, in the third chart from the top. Included on this chart are the average values of intensity of magnetization derived from the measurements of magnetization which gave the maximum value of $S$. The bottom chart in Figure 1 shows the deviation between the direction of NRM and the mean value of the direction of magnetization derived from the directions of magnetization giving the maximum value of $S$. As is to be expected, the value of this deviation is inversely correlated with the maximum value of $S$.

By interpolating between demagnetizing fields it is possible to estimate the strength of the demagnetizing field which will reduce the NRM intensity by a factor of 
G. TARASIEWICZ, E. TARASIEWICZ, AND C. G. A. HARRISON

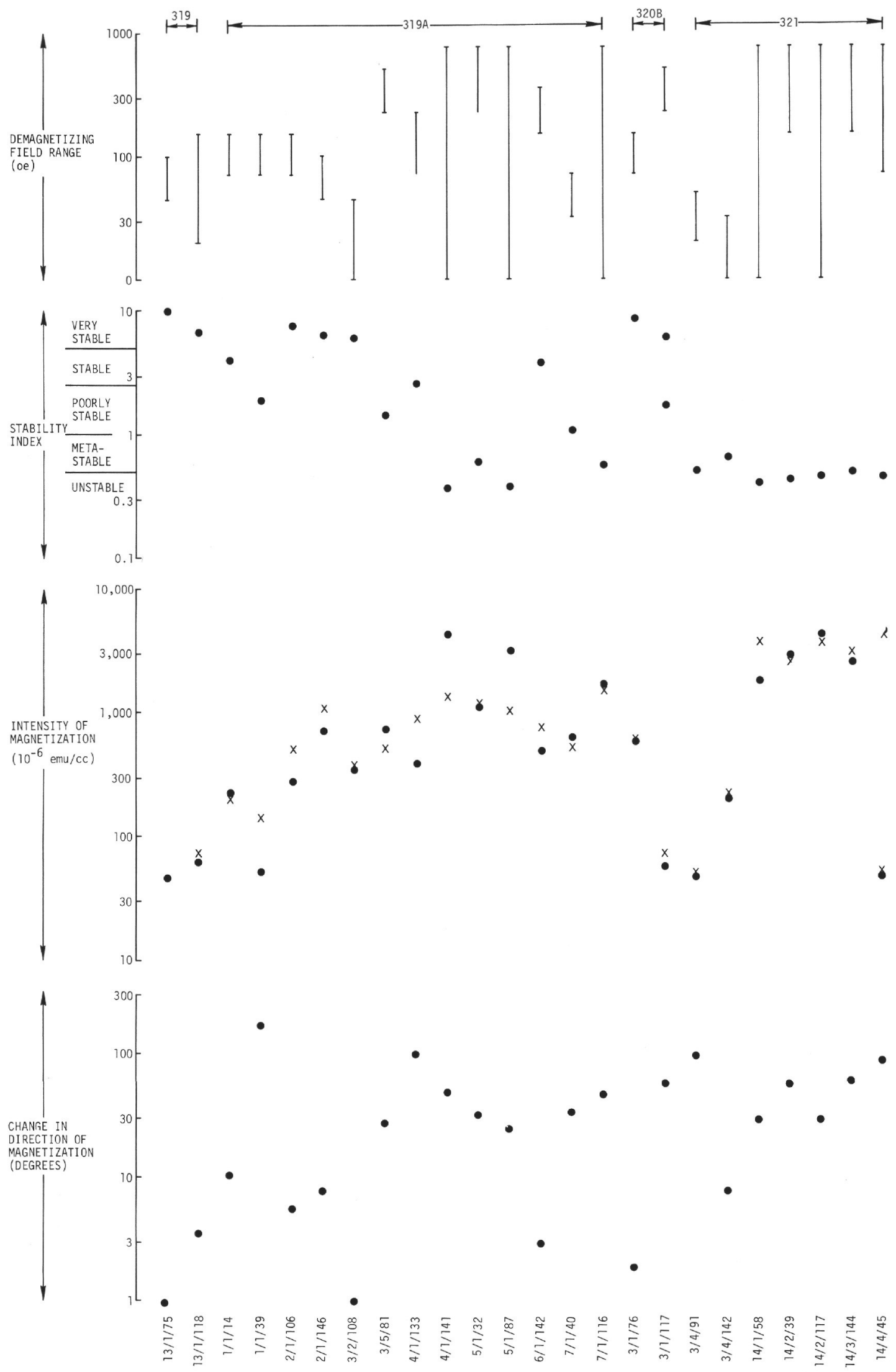

Figure 1. (Top) Demagnetizing field range for maximum value of S (ie., the stability index) (Second from top) Value of the stability index. (Second from bottom) Intensity of magnetization. The crosses are for NRM. The dots are for demagnetized intensity. (Bottom) Angular change in direcion of magnetization from NRM to maximum stability. The hole numbers are shown along the top and the sample numbers are shown along the bottom.

474 
TABLE 1

Samples Showing Much Greater Stability on Shipboard Than in This Study

\begin{tabular}{l|rc|cc}
\hline \multirow{2}{*}{$\begin{array}{c}\text { Sample } \\
\text { (Interval in cm) }\end{array}$} & \multicolumn{2}{|c|}{ Shipboard } & \multicolumn{2}{c}{ This Study } \\
\cline { 2 - 5 } & Stability & Field Range & Stability & Field Range \\
\hline 319A-5-1, 72-75 & 5.33 & $50-150$ & 0.12 & $45-150$ \\
319A-6-1, 142-145 & 4.96 & $0-200$ & 1.10 & $0-225$ \\
320B-3-1, 76-79 & 9.72 & $0-100$ & 0.94 & $0-150$ \\
320B-3-1, 117-120 & 10.75 & $0-100$ & 0.57 & $0-100$ \\
321-13-4, 91-94 & 8.06 & $0-100$ & 0.20 & $0-100$ \\
321-14-1, 58-61 & 2.97 & $0-150$ & 0.23 & $0-150$ \\
\hline
\end{tabular}

two. This is called the median destructive field. Its value is shown in the bottom portion of Figure 2, for all the samples for which it could be calculated. Many of the samples showed such a large random variation of intensity of magnetization that it was impossible to calculate a median destructive field, which has not been plotted for these samples. Sample 320B-3-1, $76 \mathrm{~cm}$ was sufficiently stably magnetized that even after a demagnetization at 750 oe, the remaining intensity was greater than $50 \%$ of the NRM, and so a median destructive field could not be established for it. Apart for this sample, all the samples for which a median destructive field could not be established are samples which give low values of the stability index.

\section{VISCOUS MAGNETIZATION}

After demagnetization at 750 oe, 10 of the samples were selected for viscous magnetization studies. They were stored in a field of 0.8 oe which was applied parallel to the axis of the cylindrical samples, and in such a way that acquisition of a viscous component of magnetization along the applied field direction was antiparallel to the component of magnetization originally present along the axis of the cylinder. The viscous component acquired was estimated by calculating the change in the component parallel to the applied field.

In most cases, a linear relationship existed between the change in the component of magnetization along the

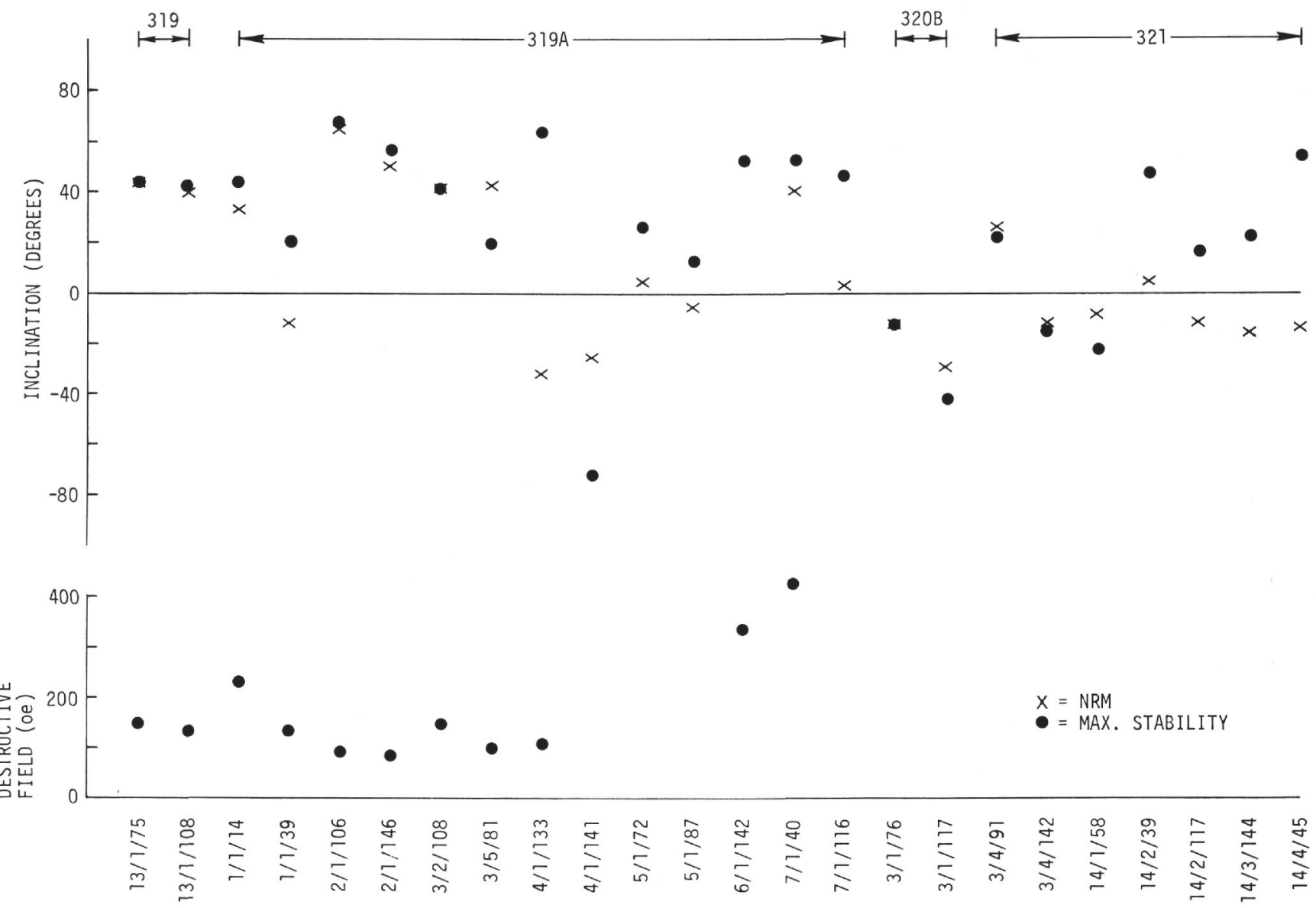

Figure 2. (Top) Inclination of direction of magnetization. NRM values are shown as crosses, and the demagnetized values are shown as dots. (Bottom) Median destructive field. The hole numbers are shown along the top and the sample numbers are shown along the bottom. 
applied field and the logarithm of time. This is illustrated in Figures 3 to 12. Two of the samples, 319-13$1,75 \mathrm{~cm}$ and $319 \mathrm{~A}-3-5,81 \mathrm{~cm}$ showed some suggestion of two different rates of acquisition of VRM, indicated by the fact that the points for the smaller times seem to follow a less steep line than do the points for the larger times. The two lines drawn on the diagrams for these two samples are the least-squares regression lines for all the points, and for the last four points, the latter being possibly the more accurate representation for the longterm acquisition of VRM. For Samples 319A-5-1, $72 \mathrm{~cm}$ and 320B-3-1, $76 \mathrm{~cm}$ the scatter of points was too large to allow a meaningful straight line to be drawn through them. In the case of Sample 320B-3-1, $76 \mathrm{~cm}$, it has already been noted that the magnetization was so stable that even after a demagnetization at 750 oe a large component of the original magnetization remained. Since the acquisition of viscous magnetization was made in the presence of this primary component, the capability

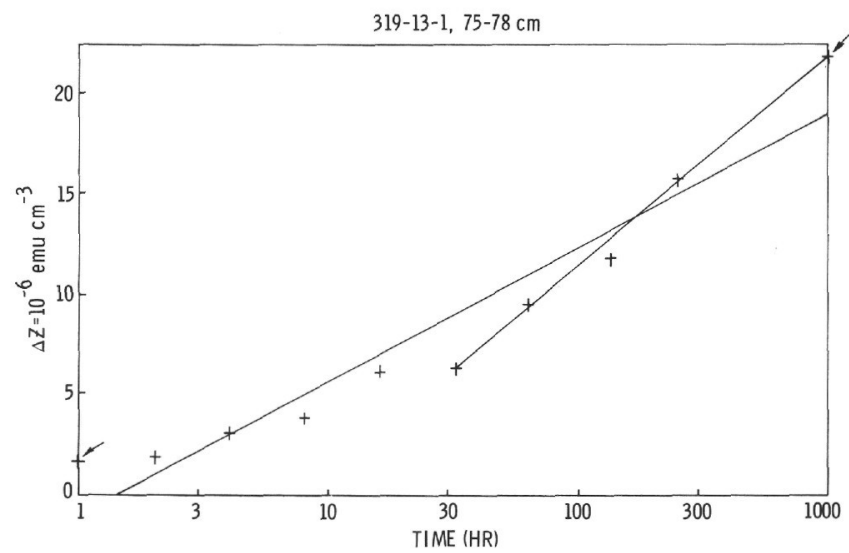

Figure 3. Acquisition of VRM plotted against the logarithm of the time in hours for ten samples.

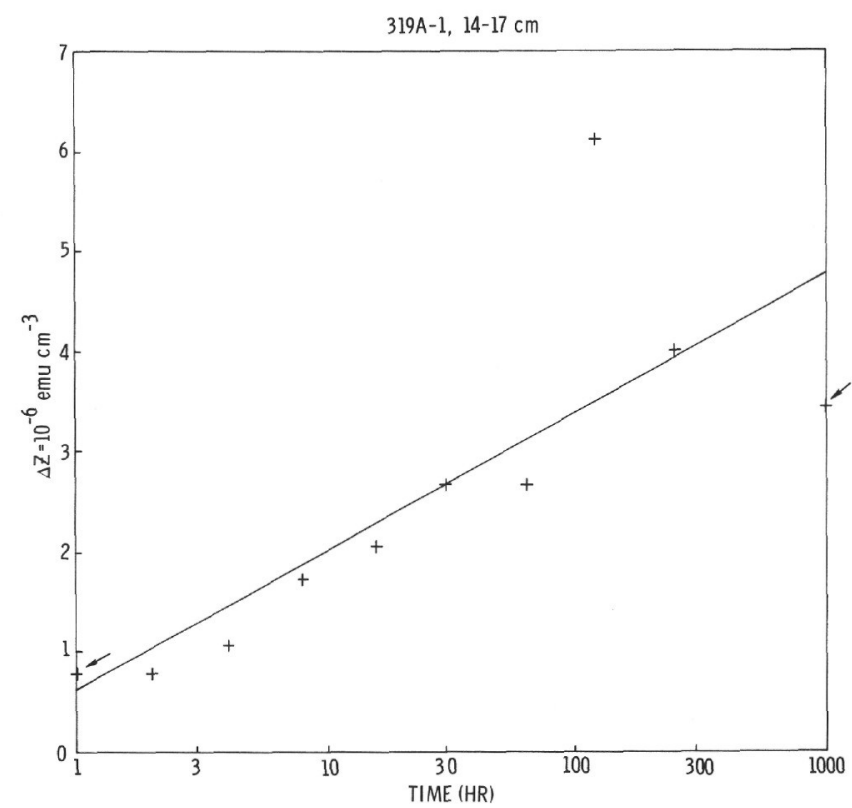

Figure 4. Acquisition of VRM plotted against the logarithm of the time in hours for 10 samples.

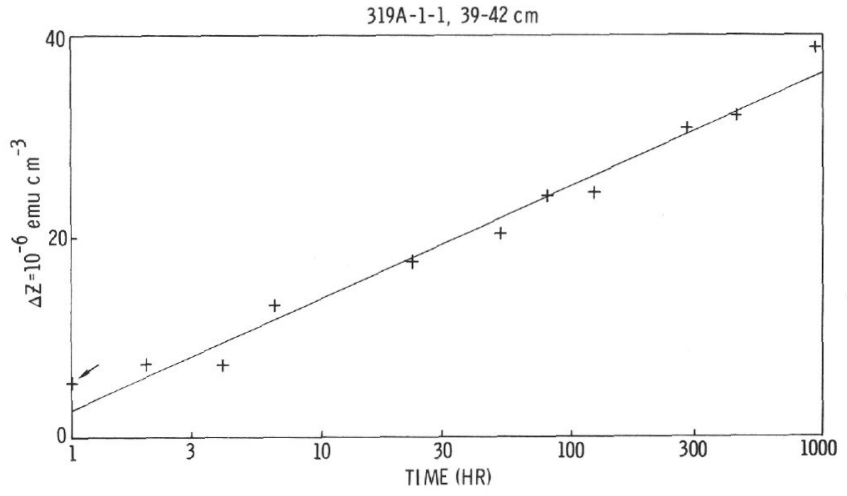

Figure 5. Acquisition of VRM plotted against the logarithm of the time in hours for 10 samples.

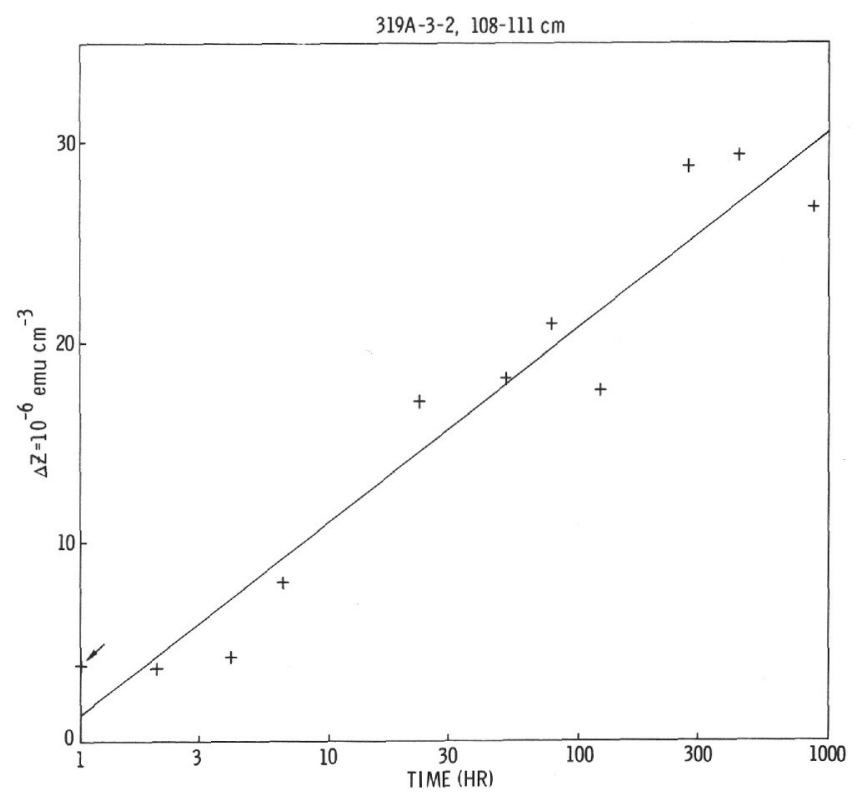

Figure 6. Acquisition of VRM plotted against the logarithm of the time in hours for 10 samples.

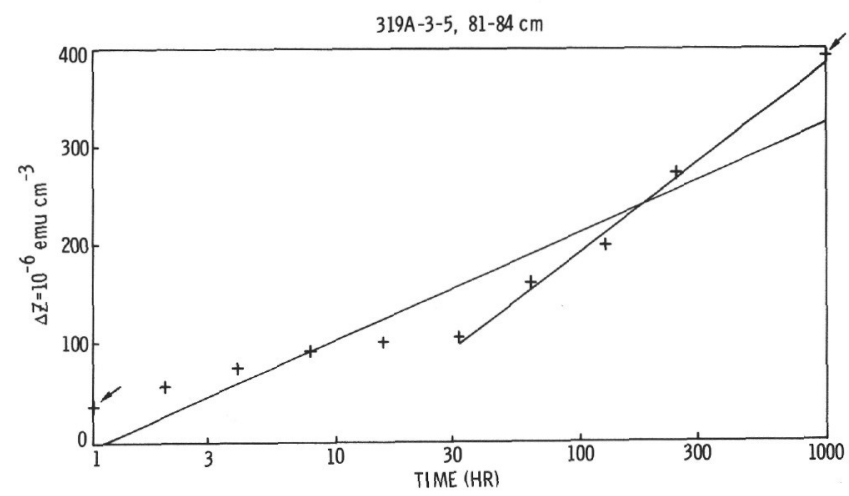

Figure 7. Acquisition of VRM plotted against the logarithm of the time in hours for 10 samples.

of detecting a small change in magnetization along one axis was not great. However, in the case of Sample $319 \mathrm{~A}-3-1,76 \mathrm{~cm}$, the total magnetization was not much greater than the component measured along the applied 
$319 \mathrm{~A}-4-1,141-144 \mathrm{~cm}$

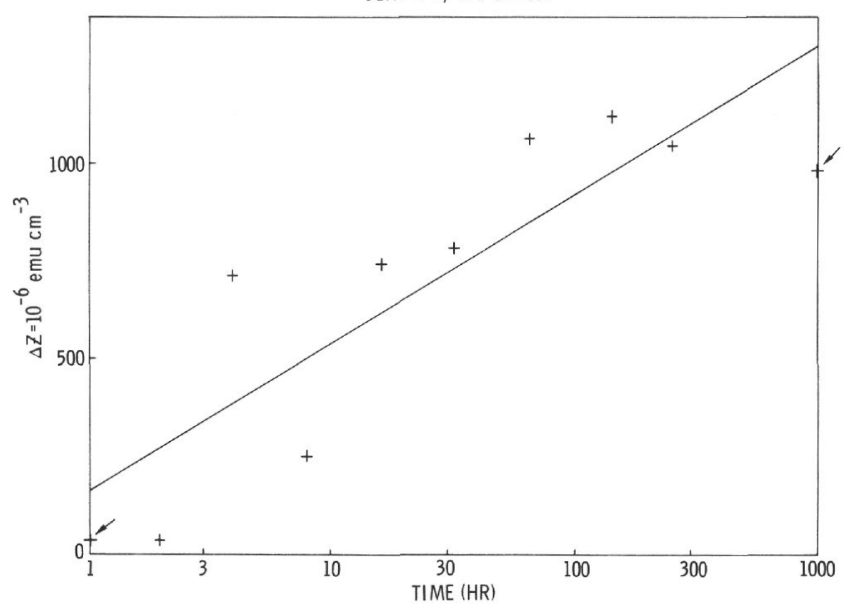

Figure 8. Acquisition of VRM plotted against the logarithm of the time in hours for 10 samples.

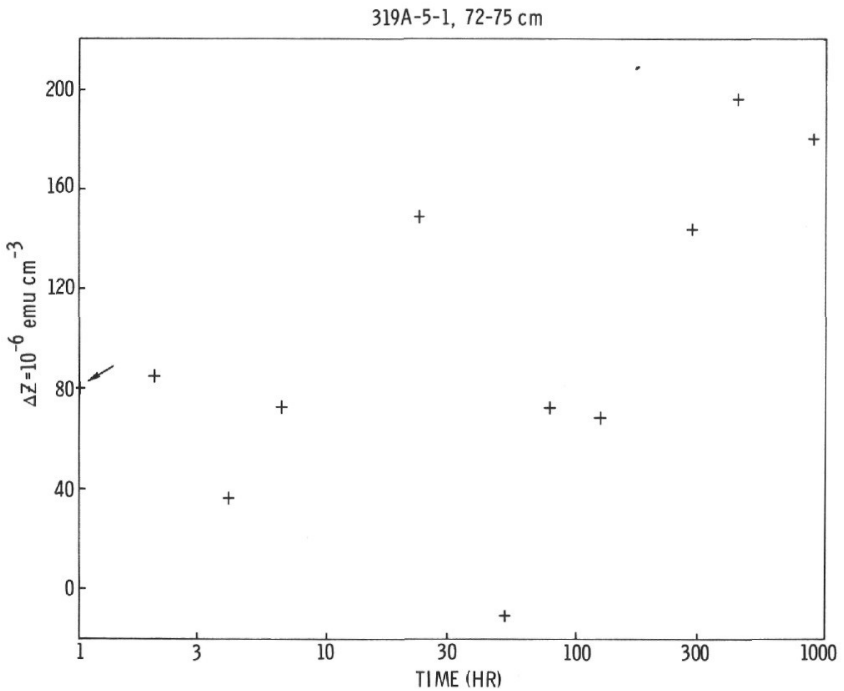

Figure 9. Acquisition of VRM plotted against the logarithm of the time in hours for 10 samples.

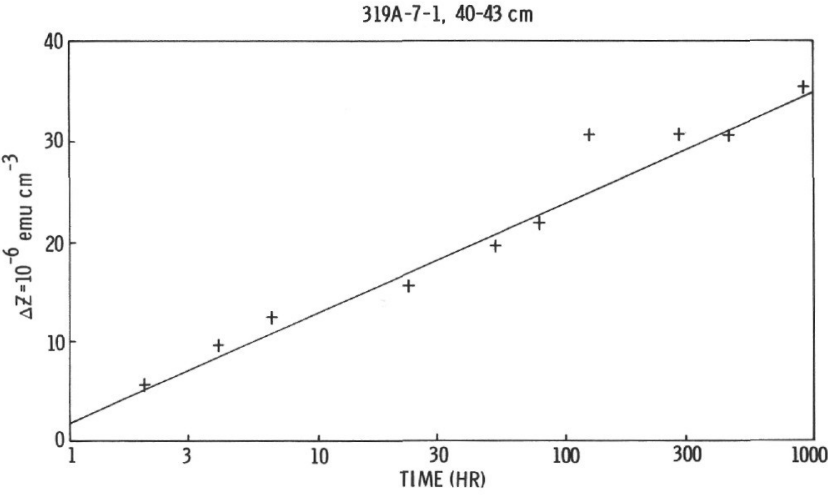

Figure 10. Acquisition of VRM plotted against the logarithm of the time in hours for 10 samples.

field, and so the scatter in this diagram must be caused by extremely unstable magnetization, producing changes before and during measurement.

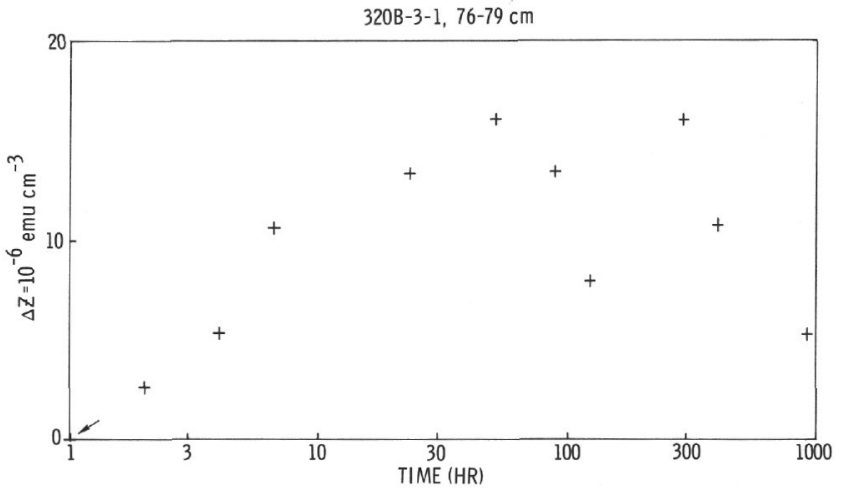

Figure 11. Acquisition of VRM plotted against the logarithm of the time in hours for 10 samples.

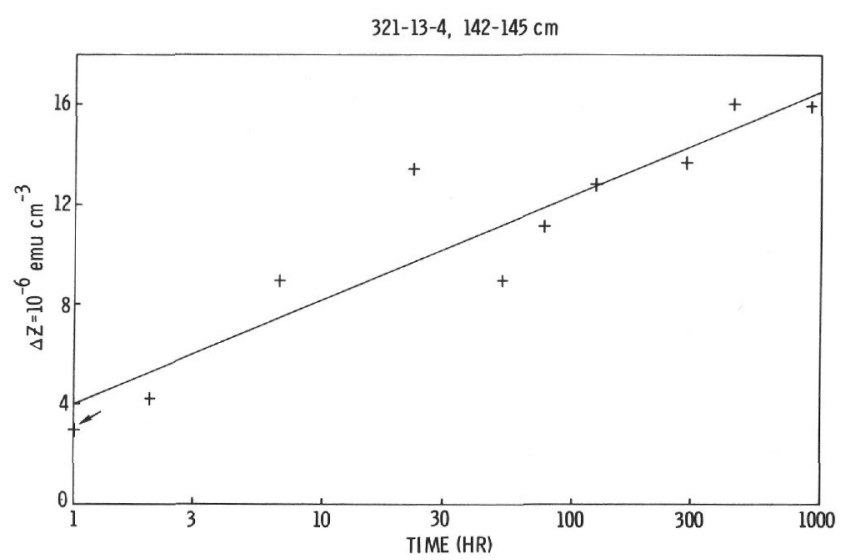

Figure 12. Acquisition of VRM plotted against the logarithm of the time in hours for 10 samples.

Much of the scatter in the other diagrams can be explained by the fact that the viscous component was being measured in the presence of other components of magnetization. For instance, Sample 319A-3-2, $108 \mathrm{~cm}$ had a total magnetization of about $140 \mathrm{micro}$ emu/cc, and hence the small deviations of less than 5 micro emu/cc of individual points from the straight line represent an error of less than $4 \%$ in the measurement of the total magnetization.

By assuming that the viscous component increases at a constant rate compared to the logarithm of time, we can estimate the amount of time necessary for the sample to have acquired all of its originally measured magnetization (or NRM) by viscous magnetization in the 0.8 -oe field. This time would of course be larger in the somewhat smaller fields encountered on the earth's surface. These times are shown in Table 2. For comparison, Table 2 also includes the stability index and the median destructive field (where it could be determined) for the 10 samples examined for the acquisition of viscous magnetization. The second set of results for Samples 319-13-1, $75 \mathrm{~cm}$ and 319A-3-5, $81 \mathrm{~cm}$ are for the larger rate of increase of VRM determined from the last four points in the relevant graphs (Figures 3 and 7).

\section{DISCUSSION}

The values of the slope in Table 2 can be compared with those found by Lowrie (1973) for dolerites from the 
TABLE 2

Times Necessary for Samples to Have Acquired all of Their

Originally Measured Magnetization (or NRM) by Viscous

Magnetization in the 0.8-oe Field

\begin{tabular}{lccccc}
\hline $\begin{array}{c}\text { Sample } \\
\text { (Interval in cm) }\end{array}$ & $\begin{array}{c}\text { Stability } \\
\text { Index }\end{array}$ & $\begin{array}{c}\text { NRM } \\
\text { Intensity } \\
10^{-6} \mathrm{emu} / \mathrm{cc}\end{array}$ & $\begin{array}{c}\text { VRM } \\
\text { Slope }\end{array}$ & $\begin{array}{c}t_{\mathrm{NRM}} \text {, b } \\
\text { years }\end{array}$ & $\begin{array}{c}\text { Median } \\
\text { Destructive } \\
\text { Field (oe) }\end{array}$ \\
\hline 319-13-1, 75 & 10.0 & 48 & 6.66 & $2.64 \times 10^{3}$ & 144 \\
& & 192 & 10.39 & $3.84 \times 10^{1}$ & \\
319A-1-1, 14 & 3.9 & 2.76 & $1.14 \times 10^{65}$ & 230 \\
319A-1-1, 39 & 1.9 & 132 & 11.22 & $3.71 \times 10^{7}$ & 135 \\
319A-3-2, 108 & 5.7 & 365 & 9.64 & $5.75 \times 10^{33}$ & 143 \\
319A-3-5, 81 & 1.4 & 530 & 109.0 & $8.42 \times 10^{0}$ & 97 \\
& & & 191.7 & $6.27 \times 10^{-1}$ & \\
319A-4-1, 141 & 0.37 & 1230 & 379.2 & $7.89 \times 10^{-2}$ & I \\
319A-5-1, 72 & 0.60 & 1150 & I & I & I \\
319A-7-1, 40 & 1.2 & 531 & 10.98 & $1.73 \times 10^{44}$ & 428 \\
320B-3-1, 76 & 8.5 & 620 & I & I & I \\
321-13-4, 142 & 0.69 & 210 & 4.18 & $2.09 \times 10^{45}$ & I \\
\hline
\end{tabular}

$\mathrm{a}_{\mathrm{T}}$ The value of $b$ in the equation $I_{\mathrm{VRM}}=a+b \log { }_{\mathrm{o}} t$, where $t$ is measured in hours and $I_{\mathrm{VRM}}$ is measured in $10^{-6} \mathrm{emu} / \mathrm{cc}$.

$\mathrm{b}_{t_{\mathrm{NRM}}}$ is the time in years taken to achieve the NRM intensity by viscous magnetization according to the above equation.

$\mathrm{c}_{\text {I means indeterminate value. }}$

Caroline Ridge in the western Pacific. His values, expressed in the same units as in Table 2 range from 280 to 390. It can be seen that in general the values obtained in this study are much less than those obtained by Lowrie. It is also true that in some cases the NRM intensity cannot be explained by the acquisition of VRM since the last definite reversal at $0.69 \times 10^{6}$ years ago, even in samples which appear to be unstable, such as 319A-1-1, $39 \mathrm{~cm}, 319 \mathrm{~A}-7-1,40 \mathrm{~cm}$ and $321-13-4,142 \mathrm{~cm}$.

There is little evidence of any correlation between the stability index, the value of the median destructive field and the time necessary to acquire the NRM intensity ( $t$ NRM) shown in Table 2. Further work is needed to determine the causes of the instability shown by many of the Leg 34 samples.

\section{ACKNOWLEDGMENTS}

The research was supported by the National Science Foundation.

\section{REFERENCES}

Fisher, R.A., 1953. Dispersion on a sphere: Proc. Roy. Soc., ser. A., v. 217, p. 295-305.

Lowrie, W., 1973. Viscous remanent magnetization in oceanic basalts: Nature, v. 243 , p. 27-30.

Tarling, D.H. and Symons, D.T.A., 1967. A stability index of remanence in paleomagnetism: Geophys. J. Roy. Astro. Soc., v. 12 , p. $443-448$. 